\title{
Rare case of non-Hodgkin's lymphoma in a patient with multiple hyperplastic colon polyps
}

\author{
Padmavathi Mali, Sudheer Muduganti, Chukwunyelu Enwezor \\ Department of Internal Medicine, Marshfield Clinic, Marshfield, Wisconsin, USA. \\ Correspondence: Padmavathi Mali. Address: Marshfield Clinic, Department of Internal Medicine, $1000 \mathrm{~N}$ Oak Ave, \\ Marshfield, WI 54449 USA. Email: mali.padmavathi@marshfieldclinic.org
}

Received: September 11, 2014

Accepted: October 20, $2014 \quad$ Online Published: December 4, 2014

DOI : 10.5430/crim.v2n1p83

URL: http://dx.doi.org/10.5430/crim.v2n1p83

\begin{abstract}
Colon polyposis, such as familial adenomatous polyposis, Puetz-Jegher's Syndrome, mutY homolog-associated polyposis, and hyperplastic/serrated polyposis syndrome, have been linked to several different types of cancer, including cancers of the gastrointestinal tract, thyroid, liver, central nervous system, genital tract, breast, pancreas, bone, sebaceous glands, ovaries, bladder, and skin. However, to date there have been no reports of an association between any of the aforementioned types of colon polyposis and lymphoma. Here, we report the case of a 79-year-old man with multiple hyperplastic polyps in the colon who was diagnosed with diffuse large B cell lymphoma of the pelvic bone. Future reporting of such associations and follow-up genetic testing is necessary to determine if there is a familial association and whether steps are needed to prevent occurrence by screening.
\end{abstract}

\section{Keywords}

Colon polyps, Hyperplastic/Serrated polyposis syndrome, Cancer, Lymphoma

\section{I ntroduction}

Several types of colon polyposis have been linked to several different types of cancer, including cancers of the gastrointestinal tract, thyroid, liver, central nervous system, genital tract, breast, pancreas, bone, sebaceous glands, ovaries, bladder, and skin ${ }^{[1-4]}$.

\section{Case presentation}

A 79-year-old Caucasian male presented to our facility with complaints of right hip pain and an inability to bear weight on his right leg. The symptoms progressed over a period of 1 month and had been accompanied by a 10 pound weight loss as a result of poor appetite. He had underlying type 2 diabetes mellitus, which was well-controlled, and a history of basal cell carcinoma, multiple seborrheic keratosis, and benign prostatic hyperplasia. The patient lived in Arizona for many years until a recent move to the midwestern United States. He was an ex-smoker, having quit 4 years ago after smoking for approximately 60 years and reported drinking alcohol on rare occasions. He had no hazardous occupational exposure. Family history was relevant for a sister who had cancer, type unknown, in her 50s and a mother who had colon cancer at age 90 . 
The patient's medical history was relevant for hyperplastic/serrated polyposis syndrome (HPS) with removal of 17 polyps this year, 8 last year, and 19 2-years prior. Biopsy of all polyps showed hyperplastic polyps and tubular adenomas. The patient was advised to undergo genetic testing but refused.

The patient was evaluated by his primary care provider and referred to our hospital for further evaluation after pelvic and hip radiography showed a lytic bone lesion in the ischium of his right pubis ramus. Examination was remarkable for tenderness on palpation of his right pelvic area with absent range of motion in his right hip. He also had multiple, raised, hyperpigmented lesions of different sizes representing seborrheic keratosis, biopsy of which was suggestive of acanthoma. One lesion on his distal left leg represented a basal cell carcinoma.

The patient's complete blood count revealed normocytic anemia with a hemoglobin of $7.8 \mathrm{~g} / \mathrm{dL}$, representing a significant drop of $3.6 \mathrm{~g} / \mathrm{dL}$ compared to his most recent complete blood count 1-month prior, and a mean corpuscular volume of 98.0 fL. He did not give any indication of acute blood loss. Other laboratory measures, including reticulocyte count, iron, total iron binding capacity, ferritin, haptoglobin, and carcionembryonic antigen were within the normal range. Treatment for anemia included transfusion of two units of packed red blood cells and continuous close monitoring of hemoglobin, which remained stable.

The patient underwent a positron emission tomography/computed tomography scan, which showed a large destructive mass in the right hemi-pelvis involving the superior ramus of the pubis extending into the acetabulum, the ilium, the ischium, and a portion of the inferior ramus of the pubis. The destructive lesion was associated with a large soft tissue mass and had a maximal standard uptake value of fluorodeoxyglucose $\left({ }^{18} \mathrm{~F}-\mathrm{FDG}\right)$ of 23.9 , consistent with malignancy. An additional lesion representing metastasis was found in the right lateral chest wall at the level of the sixth and seventh ribs.

Biopsy of the pelvic mass showed malignant cells derived from diffuse large B cell lymphoma. Immunoperoxidase staining indicated CD20- and CD3-positive, CD30- and CD138-negative tumor cells that stained roughly 70\% positive for MIB-1 with few background T cells. A bone marrow biopsy performed during work-up for anemia showed marrow that was hypercellular for age with ring sideroblasts, most compatible with low-grade myelodysplastic syndrome, but no large cell lymphoma was identified. Flow cytometry revealed a small B-cell population lacking surface light chain expression consistent with the patient's recently diagnosed large B-cell lymphoma involving bone and also showed dyserythropoiesis consistent with a diagnosis of low-grade myelodysplastic syndrome (refractory anemia with ring sideroblasts). The hematology/oncology service was consulted and the patient continues treatment on a rituximab, cyclophosphamide, doxorubicin hydrochloride, vincristine sulfate, prednisone (R-CHOP) regimen without any significant side effects.

\section{Discussion}

In the literature, cancer has been associated with several different types of colon polyps, including classic familial adenomatous polyposis (FAP), Peutz-Jeghers Syndrome (PJS), and mutY homolog (MUTYH) associated polyposis (MAP) ${ }^{[1-3]}$. Classic FAP is characterized by the presence of 100 or more adenomatous colorectal polyps and usually occurs from 20-40 years of age. Patients with FAP may have duodenal polyps, gastric polyps, and/or several extraintestinal malignancies, including follicular or papillary thyroid cancer (2\%-3\% risk), hepatoblastoma (1\% risk), and central nervous system tumors (e.g., medulloblastomas) $\left(<1 \%\right.$ risk) ${ }^{[1]}$. Genetic testing for a germline mutation in the APC gene is required for a definitive diagnosis of $\mathrm{FAP}^{[5]}$. PJS is characterized by the presence of multiple pigmented spots on the lips and the buccal mucosa and multiple gastrointestinal hamartomatous polyps of colon, small intestine, and stomach. Patients inherit PJS in an autosomal dominant fashion and are at high risk of genital tract, breast, and pancreatic cancers ${ }^{[2]}$. MAP is an autosomal recessive polyposis syndrome associated with the MUTYH gene ${ }^{[3]}$, and is also called recessive hereditary colon cancer syndrome. MAP has been associated with gastroduodenal polyps, duodenal carcinomas, osteomas, sebaceous gland tumors, and cancers of the breast, ovaries, bladder, and skin ${ }^{[3]}$. 
In the patient presented here, HPS is the condition that resulted in multiple hyperplastic/serrated colorectal polyps. The risk of colorectal cancer is increased by $50 \%$ in patients with HPS ${ }^{[4]}$, necessitating close follow-up.

Colon polyps are associated with many extraintestinal manifestations and different kinds of cancer. However, to date there has been no reported association of any of the polyposis types mentioned above with lymphoma.

In conclusion, there is a reported association of colon polyposis, such as familial adenomatous polyposis, Peutz-Jeghers Syndrome, mutY homolog-associated polyposis, and HPS with different kinds of cancer, but this case report is unique in showing an association of hyperplastic colon polyps with non-Hodgkin's lymphoma. Future reporting of such associations, if any, is necessary to determine if there is any genetic/familial association and to determine steps needed to prevent the occurrence by screening family members.

\section{References}

[1] Vasen HF, Möslein G, Alonso A, et al. Guidelines for the clinical management of familial adenomatous polyposis (FAP). Gut. 2008; 57(5): 704-13. PMid:18194984 http://dx.doi.org/10.1136/gut.2007.136127

[2] Giardiello FM, Brensinger JD, Tersmette AC, et al. Very high risk of cancer in familial Peutz-Jeghers syndrome. Gastroenterology. 2000; 119(6): 1447-53. PMid:11113065 http://dx.doi.org/10.1053/gast.2000.20228

[3] Sieber OM, Lipton L, Crabtree M, et al. Multiple colorectal adenomas, classic adenomatous polyposis, and germ-line mutations in MYH. N Engl J Med. 2003; 348(9): 791-9. PMid:12606733 http://dx.doi.org/10.1056/NEJMoa025283

[4] Miwata T, Hiyama T, Oka S, et al. Clinicopathologic features of Japanese hyperplastic/serrated polyposis syndrome. J Gastroenterol Hepatol. 2013; 28(11): 1693-8.

[5] National Comprehensive Cancer Network: NCCN colorectal cancer screening practice guidelines. Oncology (Williston Park). 1999; 13(5A): 152-79. PMid:10370926 